\title{
How a short pore forming peptide spans the lipid membrane
}

Mikkel Vestergaard, Mikkel Christensen, Sara K. Hansen, Dennis Grønvall, Lisbeth R. Kjølbye, Thomas Vosegaard, and Birgit Schiøtt

Citation: Biointerphases 12, $02 \mathrm{D} 405$ (2017); doi: 10.1116/1.4982642

View online: http://dx.doi.org/10.1116/1.4982642

View Table of Contents: http://avs.scitation.org/toc/bip/12/2

Published by the American Vacuum Society

\section{Articles you may be interested in}

Identifying the selectivity of antimicrobial peptides to cell membranes by sum frequency generation spectroscopy

Biointerphases 12, 02 D406 (2017); 10.1116/1.4982710

Orientation and characterization of immobilized antibodies for improved immunoassays (Review)

Biointerphases 12, 02D301 (2017); 10.1116/1.4978435

Predicting the orientation of protein G B1 on hydrophobic surfaces using Monte Carlo simulations

Biointerphases 12, $02 \mathrm{D} 401$ (2016); 10.1116/1.4971381

Mg-corrosion, hydroxyapatite, and bone healing

Biointerphases 12, 02 C407 (2017); 10.1116/1.4982601

Imaging XPS and photoemission electron microscopy; surface chemical mapping and blood cell visualization Biointerphases 12, 02 C408 (2017); 10.1116/1.4982644

Application of advanced sampling and analysis methods to predict the structure of adsorbed protein on a material surface

Biointerphases 12, 02D409 (2017); 10.1116/1.4983274

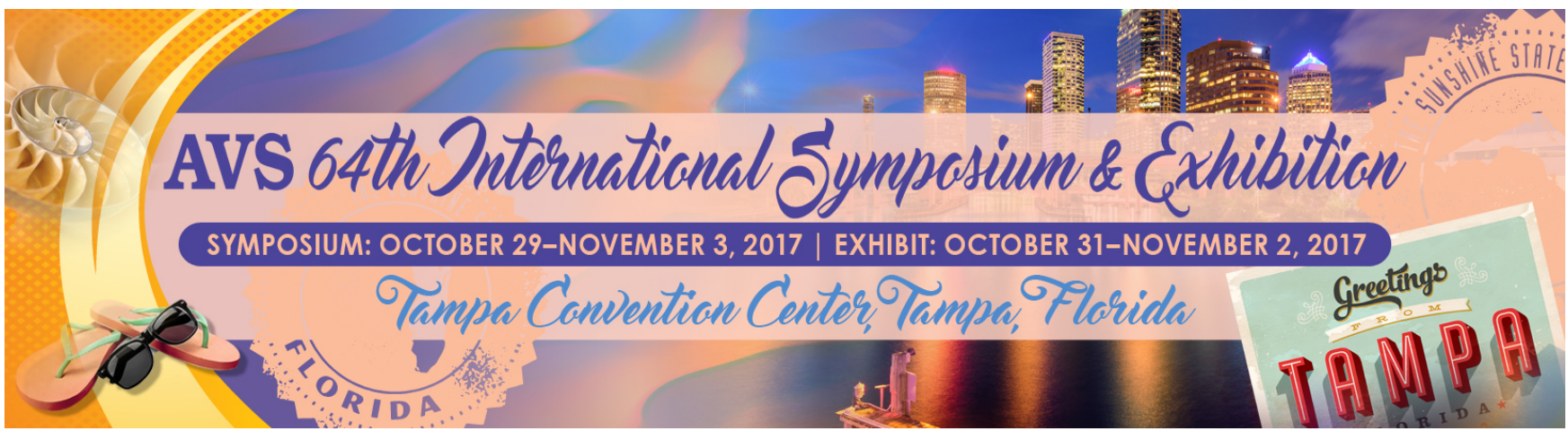




\title{
How a short pore forming peptide spans the lipid membrane
}

\author{
Mikkel Vestergaard \\ Interdisciplinary Nanoscience Center (iNANO), Department of Chemistry, Aarhus University, \\ Langelandsgade 140,DK-8000 Aarhus C, Denmark \\ Mikkel Christensen \\ Interdisciplinary Nanoscience Center (iNANO), Department of Chemistry, Aarhus University, Langelandsgade \\ 140, DK-8000 Aarhus C, Denmark and Sino-Danish Center for Education and Research, Zhongguancun \\ College, 271 N 4th Ring Road, Haidian, 100080 Beijing, China
}

Sara K. Hansen and Dennis Grønvall

Danish Center for Ultrahigh-Field NMR Spectroscopy, Interdisciplinary Nanoscience Center (iNANO) and

Department of Chemistry, Aarhus University, Gustav Wieds Vej 14, DK-8000 Aarhus C, Denmark

Lisbeth R. Kjølbye

Department of Chemistry, Aarhus University, Langelandsgade 140, DK-8000 Aarhus C, Denmark

\section{Thomas Vosegaard}

Danish Center for Ultrahigh-Field NMR Spectroscopy, Interdisciplinary Nanoscience Center (iNANO) and

Department of Chemistry, Aarhus University, Gustav Wieds Vej 14, DK-8000 Aarhus C, Denmark

Birgit Schiøtt ${ }^{\mathrm{a})}$

Interdisciplinary Nanoscience Center (iNANO), Department of Chemistry, Aarhus University, Langelandsgade 140, DK-8000 Aarhus C, Denmark

(Received 11 March 2017; accepted 13 April 2017; published 5 May 2017)

\begin{abstract}
Many antimicrobial peptides function by forming pores in the plasma membrane of the target cells. Intriguingly, some of these peptides are very short, and thus, it is not known how they can span the membrane, or whether other mechanisms of cell disruption are dominant. Here, the conformation and orientation of the 14-residue peptaibol SPF-5506- $\mathrm{A}_{4}$ (SPF) are investigated in lipid environments by atomistic and coarse grained molecular dynamics (MD) simulations, circular dichroism, and nuclear magnetic resonance (NMR) experiments. The MD simulations show that SPF is inserted spontaneously in a transmembrane orientation in both 1,2-dimyristoyl-snglycero-3-phosphocholine and 1-palmitoyl-2-oleoyl-sn-glycero-3-phosphocholine bilayers resulting in thinning of the bilayers near the peptides, which drives the peptide aggregation. Furthermore, the backbone conformation of the peptide in the bilayer bound state is different from that of the NMR model solved in small bicelles. These results demonstrate that mutual adaption between the peptides and the membrane is likely to be important for pore formation. (C) 2017 American Vacuum Society. [http://dx.doi.org/10.1116/1.4982642]
\end{abstract}

\section{INTRODUCTION}

As the incidence of multiresistant bacterial infections increases, the search for new antibiotics becomes more and more important. Thus, as antimicrobial peptides (AMPs), which are able to kill microbes, have been suggested as a source for new antibiotics, ${ }^{1}$ it is interesting to study how these peptides function for optimizing them for pharmaceutical use.

A vast variety of killing mechanisms have been proposed for different peptides, and this versatility indicates that there are a great number of possibilities for developing some of these peptides into drugs. Many AMPs have been proposed to function by perforating the cell membrane and are thus cytolytic. This can, e.g., happen by pore formation, such as proposed by the barrel-stave pore model [Fig. 1(a)], ${ }^{2}$ where

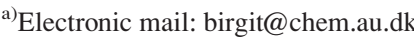

amphipathic peptides line the pore with the hydrophilic side oriented toward the pore center, or as proposed by the toroidal pore model [Fig. 1(b)], ${ }^{3}$ where both peptides and lipid head-groups line the pore. Another mechanism of membrane perforation is the carpet-model, where the peptides remain surface bound while disrupting the membrane [Fig. 1(c)]. ${ }^{4}$ These peptides are typically unstructured in solution but fold into their functional form upon membrane binding. ${ }^{5}$ Although much has been uncovered about these mechanisms, the reason for the difference between these methods is still unknown, and a better understanding of the relationship between the amino-acid sequences and the mechanisms of the cytolytic peptides is needed. ${ }^{1}$

A class of AMPs known as peptaibols is formed by soil fungi, and the presence of uncommon amino acids is a general feature of these peptides. ${ }^{6}$ Besides a high prevalence of the uncommon amino acid $\alpha$-aminoisobutyric acid (Aib), peptaibols also contain an amino alcohol at the C-terminal 
a)

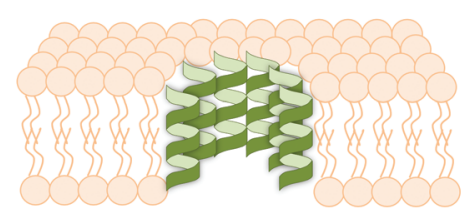

b)

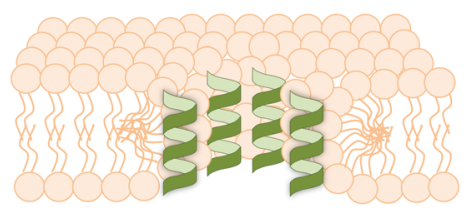

c)
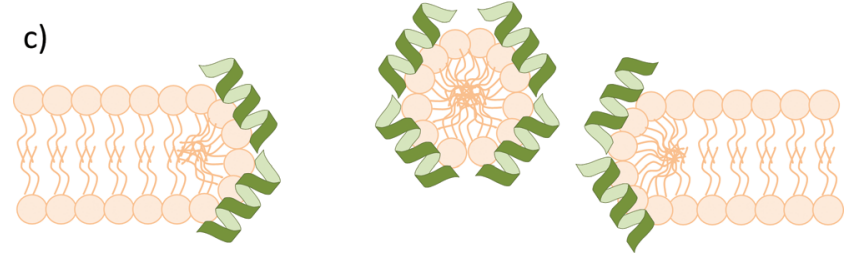

FIG. 1. Sketch of pore models: (a) Barrel-stave pore model, (b) toroidal pore model, and (c) carpet model. The peptides are illustrated as green helices, and the lipids are shown in orange.

and an acetylated $\mathrm{N}$-terminal. ${ }^{6}$ These peptides come in different lengths and are often observed to form voltage dependent ion channels in lipid membranes as exemplified by the antifungal peptide Harzianin HC (Ref. 7) and the well-studied peptaibol alamethicin. ${ }^{8}$ The peptide investigated in this study, SPF-5506- $\mathrm{A}_{4}$ (SPF), is a peptaibol with the sequence Ac-Aib 1 -Asn-Ile-Aib-Pro ${ }_{5}$-Ser-Ile-Aib-ProLeu $_{10}$-Leu-Aib-Pro-Leuol ${ }_{14}$, where Ac is an N-terminal acetylation and Leuol refers to Leucinol. This 14-residue peptide is part of a larger family of peptaibols (including, e.g., Harzianin HCs) which all have the Aib-Pro motif at positions 4-5, 8-9, and 12-13 and a conserved Asn/Gln at position $2 .^{7,9-11}$ The prolines have been suggested to contribute to the hydrophilic side of these peptides ${ }^{12}$ due to the low membrane affinity of proline, which is comparable to that of Gln and Asn. ${ }^{13}$ The hydrophilicity of proline is caused by the absent backbone hydrogen bonds related to its missing amide hydrogen and the restrained backbone dihedral angles. Thus, SPF-like peptides are expected to be amphipathic as sketched in Fig. 2.

The cytolytic ability of SPF was recently investigated, and the addition of SPF to vesicles containing fluorophores was found to result in the release of both small and large fluorophores while keeping the vesicle intact. This suggested the formation of holes with a diameter of at least $4.6 \mathrm{~nm} .{ }^{14}$ SPF is only 14 residue long, and it is unclear how these pores are formed since, assuming a helix rise of $1.5 \AA$ per amino acid, a 14-residue $\alpha$-helical peptide is too short to span the membrane; the distance between the $\mathrm{C} \alpha$ of the first and the last residue is only $\sim 19.5 \AA$, ${ }^{15}$ much shorter than the hydrophobic thickness of, e.g., a 1-palmitoyl-2-oleoyl-sn-glycero3-phosphocholine (POPC) bilayer $(\sim 28.5 \AA) .{ }^{16}$ It is thus of

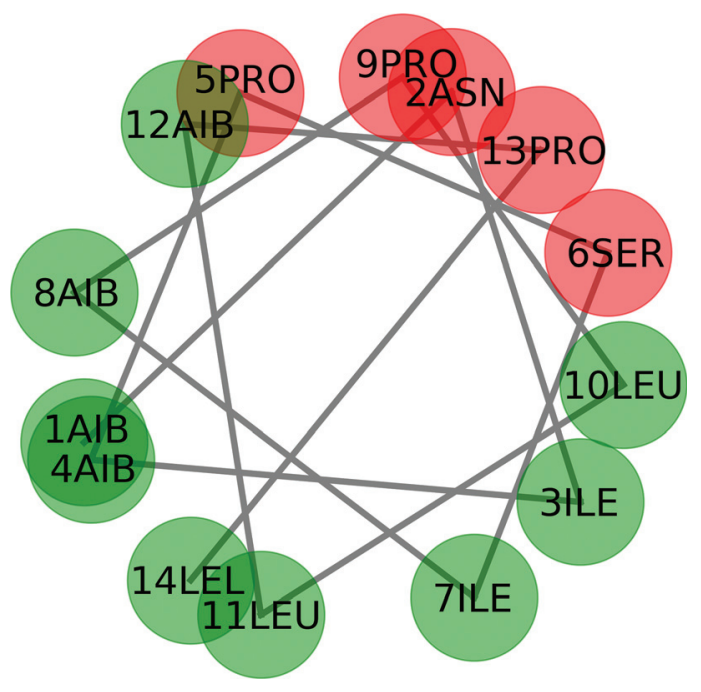

FIG. 2. Helical wheel that illustrates the amphipathic nature of SPF. The wheel is based on $\mathrm{C} \alpha$ positions projected onto the helix axis for the median structure of cluster 1 described in Sec. IV E. The residues contributing to the hydrophilic side of the peptides are shown in red, and the hydrophobic residues are shown in green. LEL abbreviates Leucinol.

great interest to investigate if and how transmembrane (TM) insertion of SPF occurs.

In the present study, we investigate the interactions between SPF and lipid bilayers by the use of all-atom (AA) and coarse-grained (CG) molecular dynamics (MD) simulations as well as circular dichroism (CD) and nuclear magnetic resonance (NMR) experiments of SPF in bicelles. With MD simulations, it is possible to study biological systems at an atomistic level at time scales from nanoseconds to microseconds and in few cases up to milliseconds. ${ }^{17}$ The fact that NMR data are directly related to the positions of the atoms with respect to each other and their environment makes it possible to back-calculate it from both the AA and CG models. ${ }^{18}$ This makes it possible to compare MD simulations both directly and indirectly with NMR by comparing the NMR data and models, respectively. ${ }^{19}$ This comparison can be both used to validate the results of the MD simulations and to provide additional structural information, inaccessible by any of the methods. Combining MD and NMR thus makes it possible to obtain further insights into biochemical systems.

\section{EXPERIMENT}

SPF was synthesized as previously described. ${ }^{14}$ A detailed description of the procedures for bicelle sample preparation, NMR, and CD experiments can be found in the supplementary material. ${ }^{44}$

\section{MODELING}

A series of atomistic MD simulations and one coarsegrained MD simulation were performed as specified in Table I. In the simulations, 64 peptides are placed on either the top 
TABLE I. MD simulations performed. All the systems contained 64 SPF peptides and 1600 lipids.

\begin{tabular}{lccc}
\hline \hline Simulation name & Peptide position & Lipid type & Simulated time $(\mu \mathrm{s})$ \\
\hline POPC $_{\text {Top64 }}$ & Top leaflet & POPC & 0.5 \\
POPC $_{\text {Both64 }}$ & Both leaflets & POPC & 0.5 \\
$P O P C_{T M 64}$ & Transmembrane & POPC & 0.5 \\
$D M P C_{T M 64}$ & Transmembrane & DMPC & 0.5 \\
$C G-D M P C_{B o t h 64}$ & Both leaflets & DMPC & 15 \\
$r v C G-D M P C_{3 \mu s}$ & Both leaflets & DMPC & 0.5 \\
$r v C G-D M P C_{15 \mu s}$ & Both leaflets & DMPC & 0.5 \\
\hline \hline
\end{tabular}

leaflet, distributed on both the leaflets, or TM as sketched for two peptides in Figs. 3(a)-3(c).

\section{A. Atomistic simulations}

The systems for the MD simulations were constructed by placing the peptides on a hexagonal grid with either a transmembrane orientation or at the bilayer surface. The peptide grid was combined with a constructed bilayer, removing three lipids from each leaflet per peptide, solvating the system, and removing water from the bilayer interior (center of bilayer $\pm 15 \AA$ ). All simulations were performed using the GROMACS 5.0.2 MD engine. ${ }^{20}$ The lipids were described using the CHARMM36 AA force field, ${ }^{21}$ while CHARMM $22^{22}$ was used for the peptides. The TIPS3P force field ${ }^{23}$ was used to describe the water molecules. The systems were minimized and simulated for $0.5 \mathrm{~ns}$ at a constant volume and temperature (NVT ensemble) with all atoms except those in the lipid tails, and the water molecule position restrained with a force constant of $1000 \mathrm{~kJ} / \mathrm{mol} \mathrm{nm}^{2}$. This was followed by $2-50 \mathrm{~ns}$ of simulations at a constant pressure and temperature (NPT ensemble) with the peptide position restrained. Finally, all restraints were removed, and the systems were minimized and equilibrated for 2 ns prior to a production run in the NPT ensemble. During equilibration, the temperature and pressure were controlled using the Berendsen weak coupling algorithm, ${ }^{24}$ while in the production run, the Nose-Hoover ${ }^{25}$ temperature coupling and the Parrinello-Rahman ${ }^{26}$ pressure control were applied. $\tau_{\mathrm{T}}$ and $\tau_{\mathrm{P}}$ were both set to $1 \mathrm{ps}$, the compressibility was set to $4.5 \times 10^{-5} \mathrm{bar}^{-1}$, and a reference temperature and pressure of $310 \mathrm{~K}$ and $1 \mathrm{bar}$, respectively, were used. Periodic boundary conditions were employed, and the neighbor list was updated by the use of the Verlet cutoff scheme with a tolerance of $5 \mathrm{~J} / \mathrm{mol} / \mathrm{ps}$. The particle mesh Ewald ${ }^{27}$ method was used to describe the electrostatic interactions with a real space cutoff of 1.2 and a Fourier spacing of $0.15 \mathrm{~nm}^{-1}$. The van der Waals (vdW) interactions were switched off between 1.0 and $1.2 \mathrm{~nm}$ by the use of force switching. ${ }^{28}$ The bonds in the water molecules were constrained using SETTLE, ${ }^{29}$ while other bond lengths involving hydrogens were constrained using a LINCS algorithm, ${ }^{30}$ thereby allowing for a time step of $2 \mathrm{fs}$.

\section{B. Coarse grained simulations}

A system with 32 peptides positioned at the surface of each leaflet of a 1,2-dimyristoyl-sn-glycero-3-phosphocholine (DMPC) bilayer was simulated for $15 \mu$ s by the use of the MARTINI 2.2 coarse grain (CG) force field. ${ }^{31,32} \mathrm{~A}$ time-step of $20 \mathrm{fs}$ was used in the $\mathrm{CG}$ simulation. In this simulation, the group cutoff scheme was applied with a neighbor list cutoff of $1.4 \mathrm{~nm}$, which was updated every tenth time step. The coulomb interactions were turned off from 0 to $1.2 \mathrm{~nm}$, while the vdW was turned off from 0.9 to $1.2 \mathrm{~nm}$ by the use of a shift function. The water molecules were modeled by the polarizable MARTINI water model, ${ }^{31,33,34}$ the dielectric constant was set to 2.5 , and the temperature was controlled by velocity rescaling ${ }^{35}$ with a coupling constant of $1.0 \mathrm{ps}^{-1}$ and a target temperature of $310 \mathrm{~K}$. After minimization, the system was equilibrated for $60 \mathrm{~ns}$ with the positions of the peptides restrained and a semi-isotropic pressure coupling controlled using a Berendsen thermostat with a coupling constant of $12 \mathrm{ps,} \mathrm{a}$ compressibility of $3 \times 10^{-4} \mathrm{bar}^{-1}$, and a target pressure of 1 bar. During the production run, the pressure was controlled using a Parrinello-Rahman ${ }^{26}$ barostat with the same coupling parameters as in the equilibration. The systems obtained at 3 and $15 \mu$ s were reverse coarse grained (rvCG) by the use of the backward tool. ${ }^{36}$ For $r \nu C G-D M P C_{3 \mu s}$ (see simulation names in Table I), one of the conformations of the SPF peptide from the end of the simulation named $D M P C_{T M 64}$ was superimposed on the reversed peptide conformations and exchanged prior to conducting the simulation to obtain peptide conformations with the correct chirality. Similarly, an end structure of that simulation was used as the starting conformation for $r v C G-D M P C_{15 \mu s}$. The equilibration process described for the atomistic systems was used hereafter.

\section{Analysis}

The median conformation of SPF in Fig. 5 was calculated as the conformation with the lowest $(\phi, \psi)$-Root mean square deviation (RMSD) compared to all other conformations. Five hundred and one snapshots distributed evenly over the full simulation $\left(P O P C_{T M 64}\right)$ were used for this analysis. Bendix for VMD was used to plot the curvature of the peptide, and default settings were applied. ${ }^{37}$ The number of inserted peptides was determined from the last frame of the simulations as the peptides in contact with the headgroup region of both leaflets. The contact was defined as any atom of the peptide within $3 \AA$ of any atom of the hydrophilic part of the lipids. The calculation of membrane thinning was conducted with g_lomepro ${ }^{38}$ in $300 \times 300$ bins per leaflet.

The match between the simulated conformations and the experimental data were calculated by a score-function for the orientation-dependent dipolar coupling and the chemical shift [Eq. (1)] and similarly for the nuclear overhauser effect (NOE) distances [Eq. (2)]. 


$$
\begin{aligned}
& \text { Score }_{\text {Orient }}\left(f_{\text {sim }}\right)=\left\{\begin{array}{cc}
\exp \left(-\ln (2) \frac{\left(f_{\text {sim }}-\left(f_{\text {exp }}-\Delta f\right)\right)^{2}}{w^{2}}\right) & f_{\text {sim }}<f_{\text {exp }}-\Delta f \\
1 & f_{\text {exp }}-\Delta f \leq f_{\text {sim }} \leq f_{\text {exp }}+\Delta f, \\
\exp \left(-\ln (2) \frac{\left(f_{\text {sim }}-\left(f_{\text {exp }}+\Delta f\right)\right)^{2}}{w^{2}}\right) & f_{\text {sim }}>f_{\text {exp }}+\Delta f
\end{array}\right. \\
& \operatorname{Score}_{\mathrm{NOE}}\left(\left\langle r_{\mathrm{sim}}\right\rangle\right)=\left\{\begin{array}{cc}
1 & \left\langle r_{\text {sim }}\right\rangle \leq r_{\exp }+\Delta r \\
\exp \left(-\ln (2) \frac{\left(\left\langle r_{\text {sim }}\right\rangle-\left(r_{\exp }+\Delta r\right)\right)^{2}}{w^{2}}\right) & \left\langle r_{\text {sim }}\right\rangle>r_{\exp }+\Delta r,
\end{array}\right.
\end{aligned}
$$

In Eq. (1) $f_{\text {sim }}$ represents the frequency for the chemical shift or dipolar coupling based on the orientation of the SPF molecule in a particular snapshot and $f_{\exp }$ is the experimentally observed resonance frequency. To allow small deviations between the simulation and experiment, we have introduced the parameters $w$ and $\Delta f$, which broaden the restraint score function. For the present calculations, we have chosen values of $w=\Delta f=5 \mathrm{ppm}$ for the chemical shift and $\mathrm{w}=\Delta f=500 \mathrm{~Hz}$ for the dipole coupling. The calculated values for the chemical shift and dipolar coupling were obtained as described elsewhere. ${ }^{39}$

The fits to the NOE distances were calculated with Eq. (2), $\left\langle r_{\text {sim }}\right\rangle$ is the calculated distance and $r_{\exp }$ is the distance obtained experimentally from the Nuclear Overhauser Effect spectroscopy (NOESY) experiment. Here, $\Delta r=w=1 \AA$.

The scores for the individual experimentally measured restraints were summed to total orientational and NOE scores. Peptides with a total orientational score of less than 5 were rejected as not being in agreement with the experiments. This cutoff is arbitrary but signifies that the score calculated using Eq. (2) already compensates largely for experimental uncertainties and hence a high value. A detailed investigation and development of a self-consistent selection of the cutoff value are beyond the scope of this work but will be presented elsewhere. The remaining structures were selected for further structural analysis. Clustering of the selected structures was performed by first aligning all peptides in the $x-y$ plane, without changing the angles with respect to the $\mathrm{z}$ axis. The aligned peptides were clustered with the gmx cluster tool of gromacs 5.1 using the single-linkage method with a $1.5 \AA$ RMSD
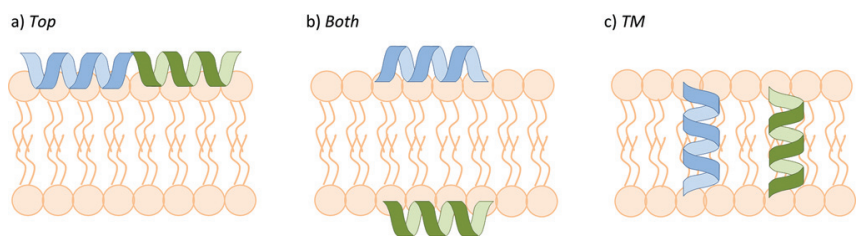

FIG. 3. Illustration of the peptide positions for two peptides: (a) all the peptides placed on the top leaflet. (b) Peptides equally distributed on both leaflets. (c) All the peptides are inserted in the transmembrane. cutoff, without further alignment of the structures. The helix tilt angles were analyzed using the gmx helixorient tool.

\section{RESULTS AND DISCUSSION}

Here, we present atomistic and coarse-grained MD simulations of SPF embedded in POPC and DMPC bilayers in both transmembrane and surface bound orientations as specified in Table I in Sec. III. These simulations were conducted to investigate the interactions between the peptide and the bilayer, and the results were compared to NMR and CD experiments conducted in isotropic micelles of DMPC and 1,2-Dihexanoyl-snglycero-3-phosphocholine (DHPC) with $\mathrm{q}=$ [DMPC]:[DHPC] $=0.5$ and alignable $\mathrm{q}=3.2$ bicelles.

\section{A. Spontaneous C-terminal insertion}

To mimic the conditions obtained when the SPF peptides are initially added to a cell or a vesicle where only the outer leaflet is exposed to peptides, an atomistic simulation was conducted with 64 SPF peptides positioned at the hydrophobic/hydrophilic interface of the top leaflet of a POPC bilayer $\left(P O P C_{T o p 64}\right)$. This causes a strain on the outer leaflet, which may be part of the reason why SPF and AMPs are translocated over the membranes. Interestingly, 22 of the 64 peptides are inserted in a transmembrane orientation without interacting with other peptides during the insertion process. Thus, the highly hydrophobic character of SPF appears to enable a transmembrane insertion in lipid membranes even though the peptide is only 14 residue long. Whether the transmembrane orientation obtained was merely an intermediate step in the process of moving the peptide from the outer leaflet to the inner leaflet was tested by embedding $32 \mathrm{SPF}$ peptides in each leaflet of a POPC bilayer $\left(P O P C_{B o t h 64}\right)$. Herein, it was found that SPF peptides from both the leaflets were observed for insertion in a transmembrane orientation (11 of 64 peptides); thus, the transmembrane orientation appears to be a stable conformation or at least a state with a long life-time. The timescale of peptide insertion and extraction is on the timescale of $10 \mathrm{~ns}$ to hundreds of nanoseconds, and the equilibrium distribution of inserted and surface bound peptides therefore cannot be reached within the timescale of these 

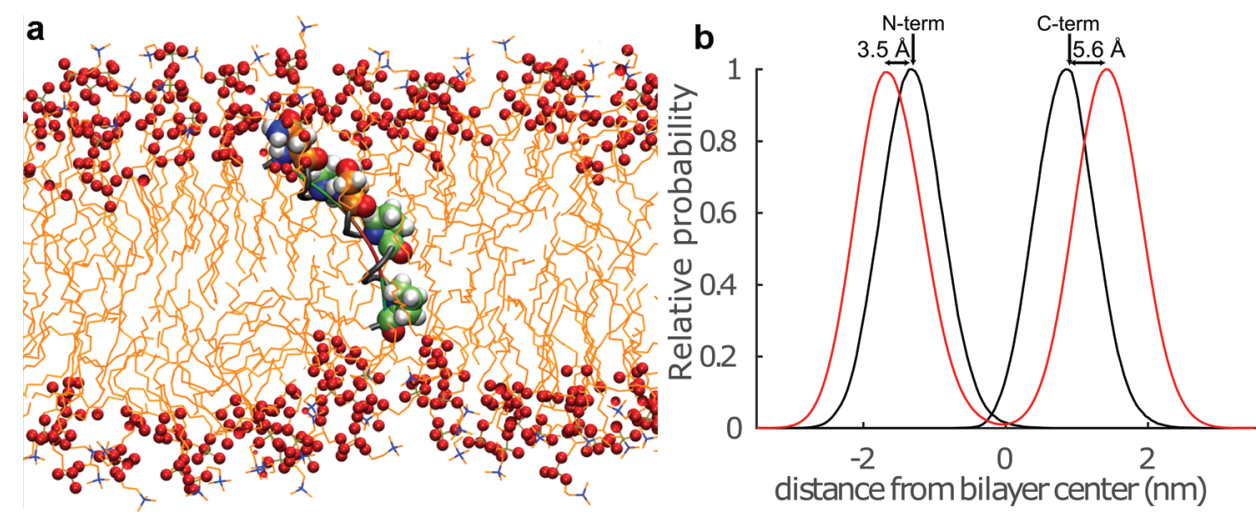

FIG. 4. (a) Illustration of how SPF is embedded in a POPC bilayer. Lipids are shown in lines with carbons colored orange, and the oxygen atoms are shown as red spheres. The peptide backbone is shown as gray ribbon, Asn2 and Ser6 are highlighted with orange carbons, and proline carbons are shown as green spheres, while all other atoms of these residues are shown in spheres with the usual atom coloring. (b) Distribution of the $\mathrm{C}_{\alpha}$ of the $\mathrm{N}$ - and $\mathrm{C}$-terminal residues of the transmembrane SPF-peptides (black) and the ester oxygens of POPC in $P O P C_{T M 64}$ (red) normalized such that the maximum value for each plot equals one.

simulations. A coarse-grained MD simulation with 32 peptides on each leaflet $\left(C G-D M P C_{\text {both } 64}\right)$ was performed to investigate the peptide insertion at a longer timescale of $15 \mu \mathrm{s}$. Here, 56 of 64 peptides were inserted into the membrane. The insertion process consistently occurred with the C-terminus being transferred over the hydrophobic part of the membrane in both the AA- and CG-simulations, which is most likely related to the higher hydrophobicity of the C-terminus compared to the N-terminal part of the peptide which contains Asn 2 and Ser6. The transmembrane orientation of the peptide was investigated further by the atomistic simulation of 64 SPF-peptides initially placed in a transmembrane orientation in a POPC bilayer $\left(P_{P O P C_{T M 64}}\right)$. Herein, most of the peptides remained in the transmembrane (56 of 64).

From visual inspection and by analysis of the $\mathrm{C} \alpha$ distribution of the transmembrane peptides along the bilayer normal (Fig. 4), it is apparent that the C-terminus of the peptide is positioned in the hydrophobic part of the membrane, $5.6 \AA$ from the median position of the ester oxygens of the lipids. The N-terminus, on the other hand, is located closer to the surface ( $3.5 \AA$ from the ester oxygens) and thus interacts significantly more with the hydrophilic part of the lipids, due to, e.g., the hydrophilic residues Asn2 and Ser6, which explains the preference for $\mathrm{C}$-terminus insertion.

\section{B. Mutual adaption of the peptides and the membrane}

The transmembrane peptides form a bent amphipathic helix with the hydrophilic residues and the prolines positioned on the convex side of the peptide and the concave side covered by hydrophobic residues [Fig. 5(a)]. The most pronounced helix curvature is observed near Pro9.

As previously mentioned, a 14-residue helix peptide is substantially shorter than the hydrophobic thickness of the membrane: to assess this mismatch, the distance between the $\mathrm{C} \alpha$ of the first and the last residue [Fig. 5(b)] was calculated to obtain a measure of the peptide length. The peptide length distribution for the peptides in $P O P C_{T M 64}$ which remained in a transmembrane orientation [red in Fig. 5(b)] peaks at $23 \AA$ which is larger than that observed for the structures in the NMR ensemble solved in $\mathrm{q}=0.5$ bicelles ${ }^{14}$ [gray in Fig. 5(b)]. This indicates that the peptides are stretched in the bilayer as compared to when it is found in $\mathrm{q}=0.5$ bicelles. A similar simulation in a DMPC bilayer $\left(D M P C_{T M 64}\right)$ was conducted to investigate the effect of the membrane thickness on the peptide length as a DMPC membrane $(\sim 25 \AA)$ is thinner than a 1,2-dioleoyl-sn-glycero-3-phosphocholine membrane $(\sim 28.5 \AA) .{ }^{16,40}$ All peptides remained in the transmembrane throughout the simulation, and the peptides were found to be of similar length [orange in Fig. 5(b)] as in a POPC bilayer and therefore independent of the membrane thickness. The peptide is thus much longer in both DMPC and POPC bilayers than a typical $\alpha$-helical peptide $(\sim 19.5 \AA)$ although shorter than a 310 -helix $(\sim 26 \AA) .{ }^{15}$ The
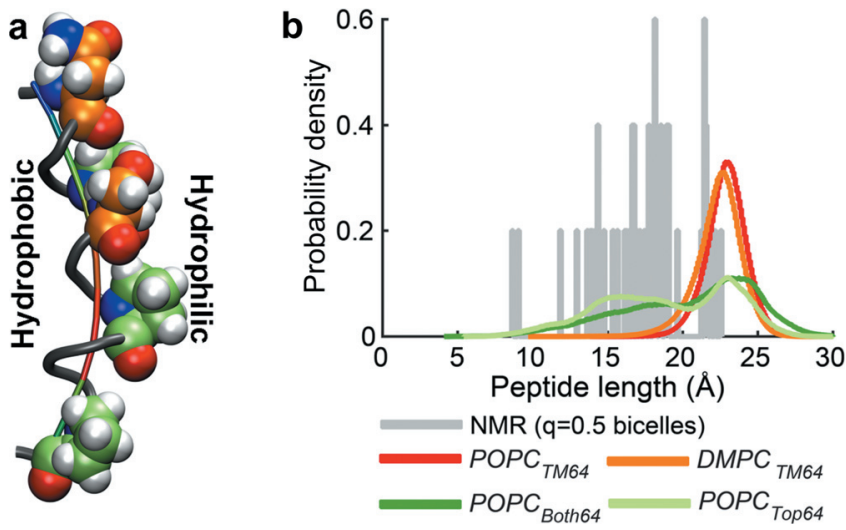

FIG. 5. Peptide curvature and length. (a) Median conformation of SPF in $P O P C_{T M 64}$. The backbone is shown as gray ribbon, Asn2 and Ser6 are highlighted with orange carbons, and proline carbons are shown as green spheres, while all other atoms of these residues are shown in spheres with the usual atom coloring. The bend of the helix is shown by the line colored according to the curvature calculated with Bendix (Ref. 37) (blue, green, and red for low, medium, and high curvatures, respectively). (b) The distribution of the distance between the $\mathrm{C} \alpha$ of the first and the last residue of SPF in the available NMR models (Ref. 14) and in the simulations. 
deviation from the latter may be due to the bending of the peptide apparent from Fig. 5(a). The length distributions for the surface bound peptides in $P O P C_{\text {Both64 }}$ and $P O P C_{T o p 64}$ are much broader than those observed for the transmembrane peptides, indicating that the peptides are highly flexible when surface bound while more fixed when they are in a transmembrane orientation [Fig. 5(b)].

Although the transmembrane peptides are stretched by the bilayer compared to those observed in the NMR models, they are still shorter than the hydrophobic thickness of both a POPC bilayer ( $\sim 28.5 \AA)$ and a DMPC bilayer $(\sim 25 \AA)$, indicating that a thinning of the bilayer is needed for the bilayer to match the length of the peptides. ${ }^{16}$ The thickness of the membrane was investigated using the g_lomepro tool, ${ }^{38}$ and the average over the last $10 \mathrm{~ns}$ of $P O P C_{T M 64}, P O P C_{B o t h 64}$, $r v C G-D M P C_{3 \mu s}$, and $r v C G-D M P C_{15 \mu s}$ is shown in Fig. 6. Significant thinning is observed near the transmembrane peptides in $P O P C_{T M 64}$ [Fig. 6(a)], which is compensated for by a thickening of the bilayer in other areas. In contrast, the surface bound peptides in $P O P C_{\text {Both64 }}$ [Fig. 6(b)] are not consistently colocalized with the area of membrane thinning. Transmembrane SPF peptides thus appear to thin the membrane to obtain a better match between the hydrophobic thickness of the membrane and the length of the peptide. The systems backmapped to an atomistic description from the coarse-grained simulation $C G-D M P C_{B o t h 64}$ at $3 \mu \mathrm{s}$ ( $r v C G$ $\left.D M P C_{3 \mu s}\right)$ and $15 \mu \mathrm{s}$ (rvCG-DMPC $15 \mu s$ ), also indicate thinning in the areas around the peptides as shown in Figs. 6(c) and $6(\mathrm{~d})$.

\section{Peptide ordering}

The longer timescale of the coarse-grained simulation revealed clustering and ordering of the peptides within the membrane. The peptides pack parallel and side-by-side in a two layer arrangement and form a band that spans the membrane [see the top view image in Fig. S1]. After $15 \mu$ s of coarse-grained simulations, four peptide aggregates were formed. All the aggregates were stable after the atomistic simulation time although, as expected, the structure was less rigid than that in the coarse-grained simulation. The increased dynamics in the atomistic simulations is most likely caused by the additional degrees of freedom and that a predetermined secondary structure is imposed in the $\mathrm{CG}$ simulation limits of the structural flexibility. The four aggregates are illustrated in Fig. 7. Most peptides are transmembrane, and the few surface bound peptides interact with the ends of some of the inserted peptides; however, their a) $P O P C_{T M 64}$

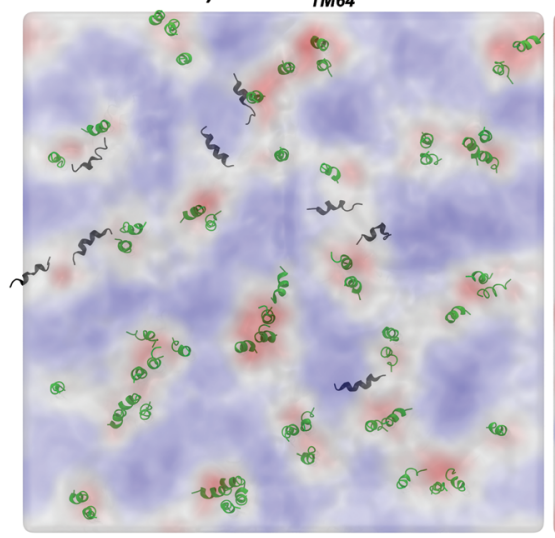

c) $r v C G-D M P C_{3 \mu s}$

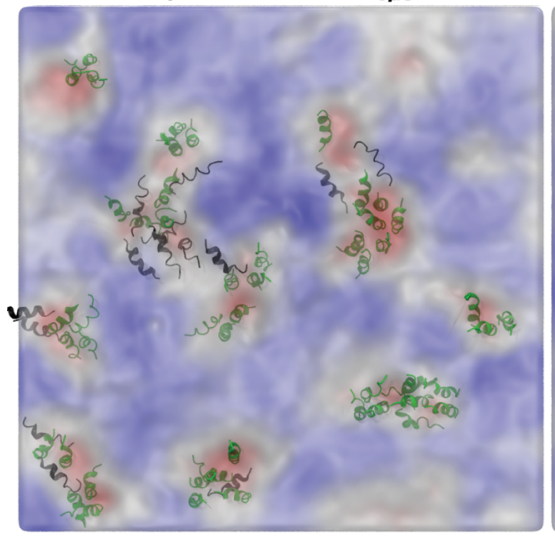

b) $P O P C_{B o t h 64}$

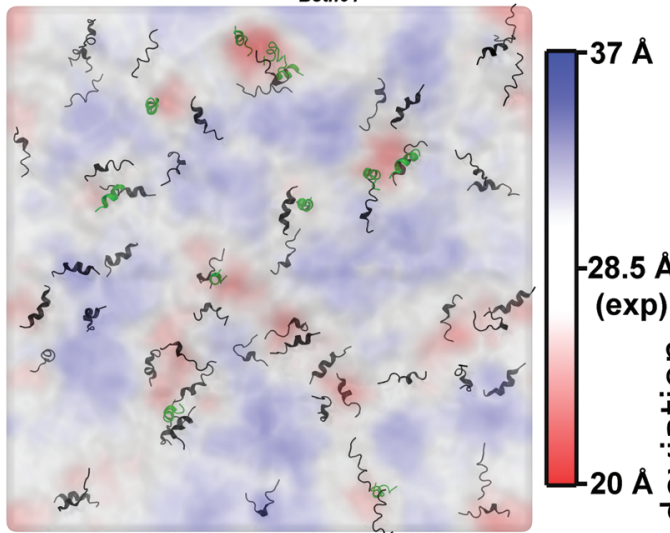

$28.5 \AA$

(exp)

$\frac{c}{0}$

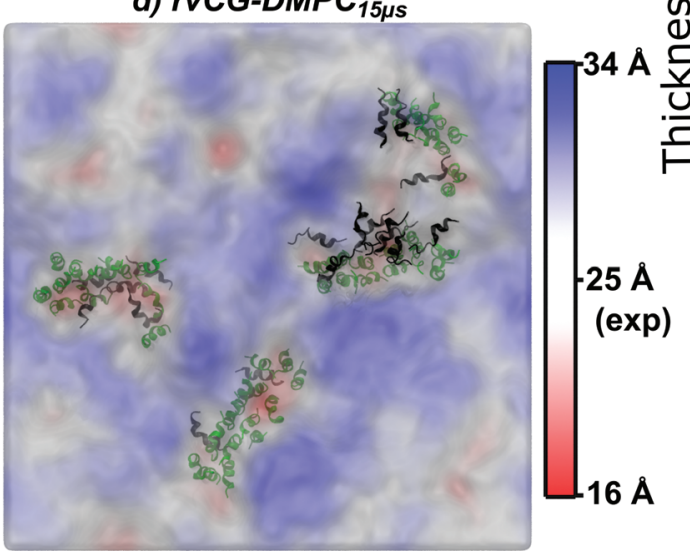

FIG. 6. Membrane thickness averaged over the last $10 \mathrm{~ns}$ of (a) $P O P C_{T M 64}$, (b) $P O P C_{B o t h 64}$, (c) $r v C G-D M P C_{3 \mu s}$, and (d) $r v C G-D M P C_{15 \mu s}$. Thickening compared to the experimental value is shown in blue, while thinning is colored red. Surface bound peptides are colored black, while transmembrane peptides are green. 
a)

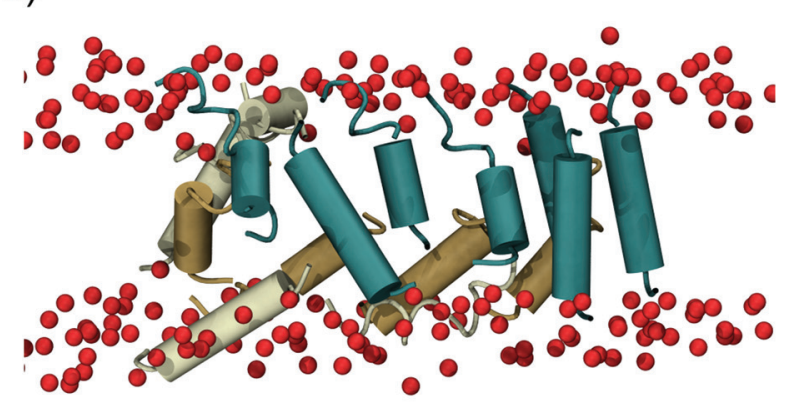

b)

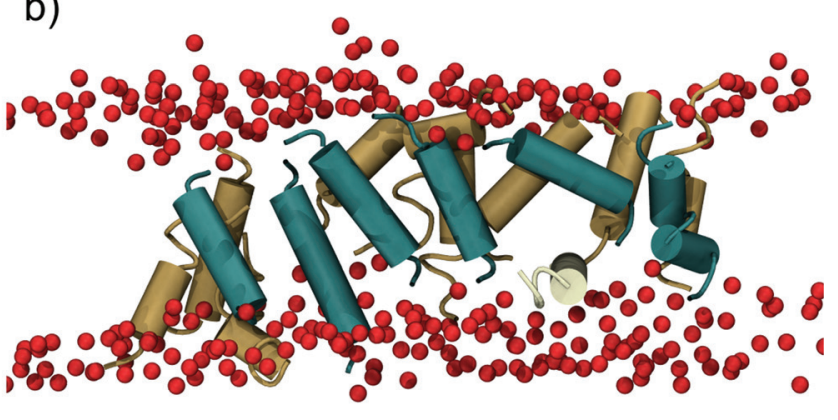

c)

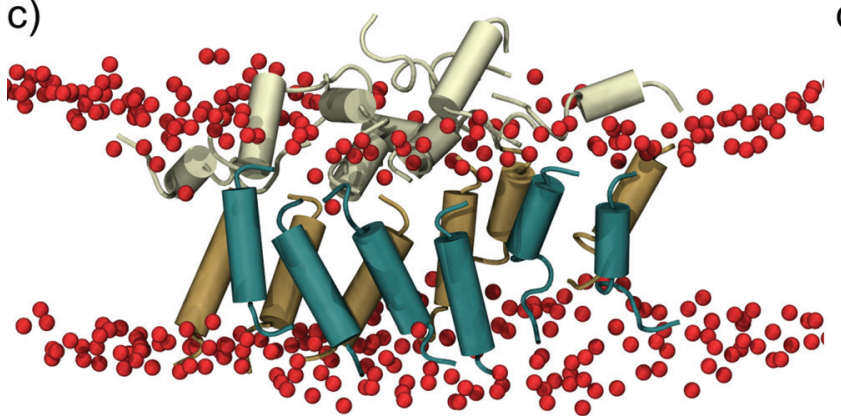

d)

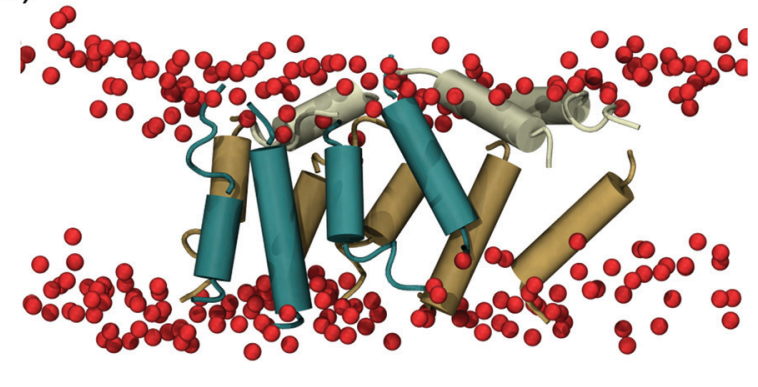

FIG. 7. Illustration of the four aggregates (a)-(d) of peptides formed in the coarse-grained simulations. The structures are from the end frame of $r v C G$ $D M P C_{15 \mu s}$. The peptides are colored based on the two layers in the two-layer arrangement of the peptides; the front layer in colored blue, and the back layer is colored brown. Surface-bound peptides and peptides not included in the arrangement are shown in white. The DMPC ester oxygens are shown as red spheres.

presence is not required for other peptides to be able to span the membrane as not all transmembrane peptides are in contact with the surface bound peptides.

\section{Peptide secondary structure}

To further investigate the conformation of SPF, the backbone angles of SPF were measured in the simulations and compared to the values in the NMR ensemble solved in $\mathrm{q}=0.5$ bicelles $^{14}$ as well as the NMR models of Harzianin HC IX solved in methanol, which show a similar amphipathic structure and only differ from the sequence of SPF by the presence of Leu3, Ala6, and Iva10 (isovaline). ${ }^{41}$ These data are plotted in Fig. 8, and it is apparent that the NMR models of SPF (light blue), including the model the simulations were initiated from (black), deviates significantly from both the transmembrane peptides in the MD-simulation (green) and the harzianin HC IX models (purple).

The difference in the backbone conformation indicates that the secondary structure is affected by changing the environment from a bilayer to $\mathrm{q}=0.5$ bicelles, which is likely to be related to the higher curvature of the membrane mimics and the increased solvent accessibility of the transmembrane peptides in $\mathrm{q}=0.5$ bicelles compared to that in a bilayer or in a $\mathrm{q}=3.2$ bicelle environment. ${ }^{42}$ The large difference in the CD spectra measured for SPF in $q=0.5$ and in the more bilayerlike $q=3.2$ bicelles supports the observation that the membrane mimic affects the secondary structure (Fig. 9). In $q=3.2$ bicelles, the $C D$ spectrum shows a shape similar to both that was observed for the peptide in 2,2,2Trifluoroethanol (TFE) (inset in Fig. 9) and that was previously published for Harzianin HC IX in methanol, indicating that these peptides assume similar conformations. ${ }^{41}$

Along the same lines, the backbone angles of the transmembrane SPF peptides in $P_{O P C} C_{T M 64}$ (green in Fig. 8) are similar to those observed for Harzianin HC IX (dark purple in Fig. 8), indicating that SPF may assume the (Xaa-Yaa-AibPro)- $\beta$-bend ribbon spiral conformation proposed for Harzianin HC IX. ${ }^{41}$ The N-terminal residues are the only part of the peptide which in the simulation shows significant deviations from the Harzianin HC IX models. This deviation can be caused by the simulations being initiated from a SPF conformation with a very different starting conformation. The largest deviation was observed in the $\varphi$-angle of residue 2 which, by investigating the angle distribution plots (Fig. S2 in the supplementary material), was found to be due to two different populations of the angle being present; one with an angle similar to the starting conformation and one similar to the Harzianin HC IX. The time evolution of the number of peptides showing the same conformation as Harzianin HC IX in $\operatorname{Asn} 2(\varphi)$ (Fig. S3 in the supplementary material) shows an increase from $0 \%$ to $\sim 35 \%$ over $500 \mathrm{~ns}$. Thus, the simulation indicates that the peptides go toward the same conformation as Harzianin HC IX solved in methanol. ${ }^{41}$ The systems are too large to obtain a time-scale where all peptides can reach equilibrium, but we assume that it would equilibrate at an Asn2 $(\varphi)$ similar to Harzianin HC IX. We therefore conclude that SPF attains the (Xaa-Yaa-Aib-Pro)- $\beta$-bend ribbon spiral conformation in a bilayerlike environment, which deviates from the structure observed in $\mathrm{q}=0.5$ bicelles. The surface bound peptides $\left(P O P C_{\text {Both64 }}\right.$ and $\left.P O P C_{\text {Top64 }}\right)$ take on similar 


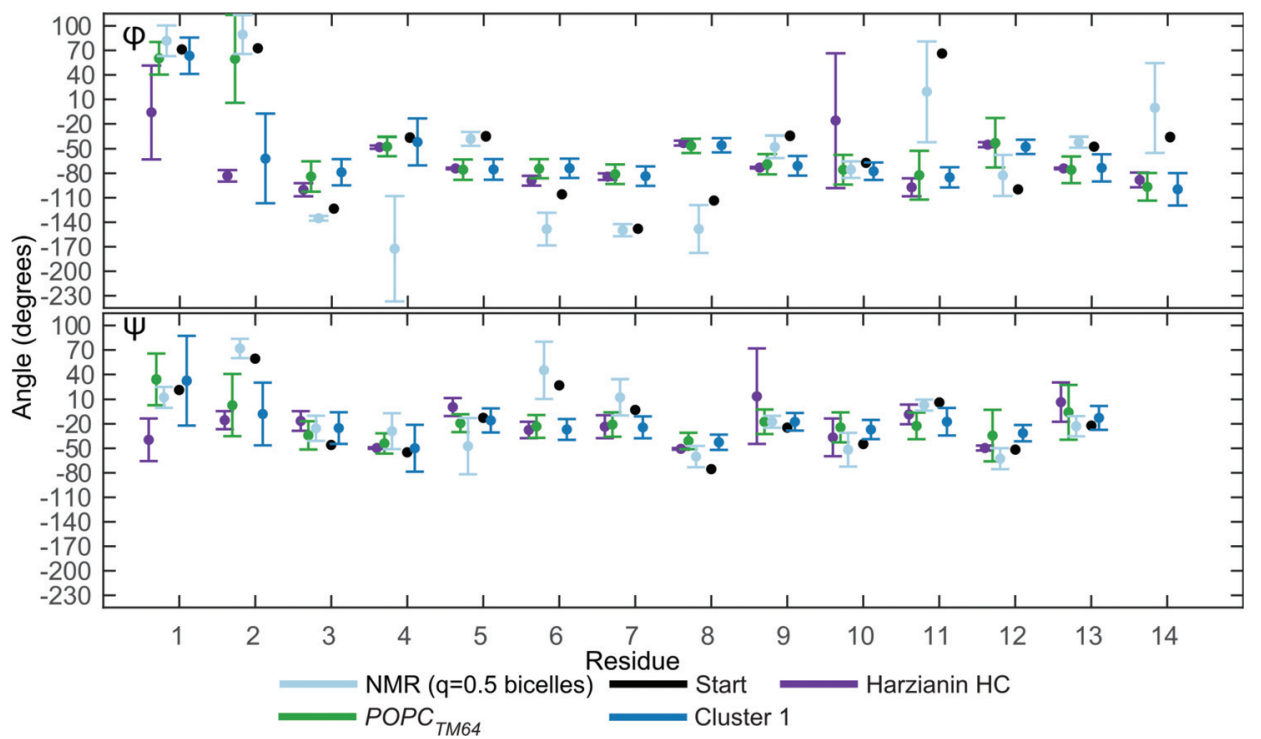

FIG. 8. Average backbone angles $\phi$ and $\psi$ of SPF including standard deviations of the distributions for each residue. The data are shown for the conformation, and the simulations were initiated from (black), for all the frames in the $P O P C_{T M 64}$ simulation (green) and in cluster 1 (dark blue, described in Sec. IVE), along with the experimental values of SPF in $\mathrm{q}=0.5$ bicelles (light blue) and Harzianin in methanol (purple).

conformations as observed for the transmembrane peptides (Fig. S4 in the supplementary material) although larger standard deviations are observed, indicating a larger conformational flexibility in the surface bound mode compared to when the peptide is in transmembrane orientation.

\section{E. Validating the transmembrane orientation}

To validate the hypothesis found above, that the transmembrane orientation is the equilibrium orientation observed in experiments, NOE distances were measured by liquid state NMR in $\mathrm{q}=0.5$ bicelles. In addition, we measured orientation-dependent ${ }^{15} \mathrm{~N}$ chemical shifts and ${ }^{1} \mathrm{H}_{-}{ }^{15} \mathrm{~N}$ dipolar couplings of ${ }^{15} \mathrm{~N}$ labeled SPF (labeled at Aib4, Aib8, and Aib12) in $\mathrm{q}=3.2$ bicelles by the use of oriented solid state NMR, yielding a total of 6 orientational constraints (see

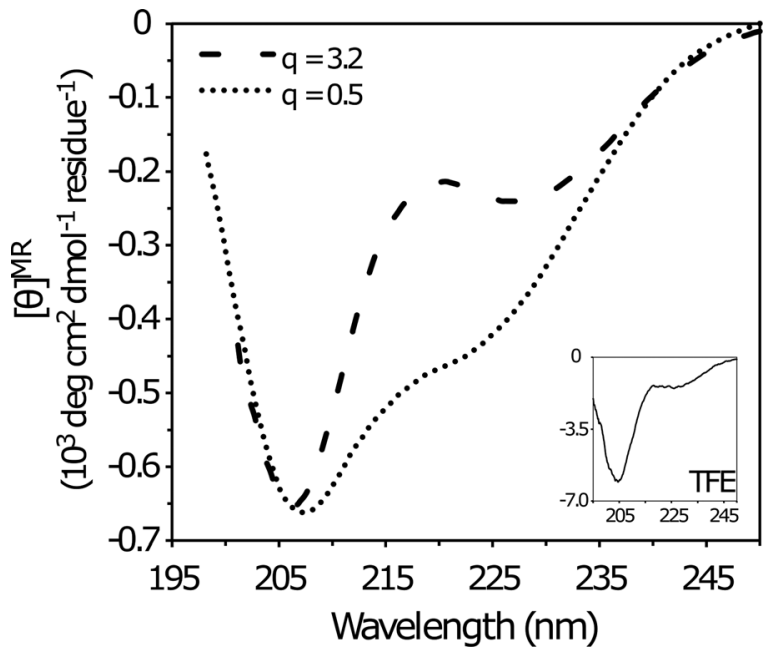

FIG. 9. CD spectrum of SPF in bicelles with different q-values. A DMPC:peptide ratio of 25 and 50 was used in $q=3.2$ and $q=0.5$ bicelles, respectively. The inset shows the CD-spectrum of SPF in TFE.
Fig. S7 in the supplementary material). All snapshots from the 6 atomistic simulations were scored according to their agreement with the experimental data as explained in Sec. III C. The 18199 peptide conformations that scored more than five of six in Score $_{\text {Orient }}$ were selected and clustered and are illustrated in Fig. 10. The selected structures are a small fraction of the total number of sampled peptide structures, as the calculated chemical shift and dipolar couplings are fluctuating, and therefore, only these structures are in agreement with five of six parameters at the same time.

This resulted in three major clusters, representing $40.7 \%$, $10.8 \%$, and $9.2 \%$ of the selected structures, respectively. The structures in all three clusters score on average $\sim 165$ of the maximum of 193 in the sum of Score $_{\mathrm{NOE}}$ as this is the amount of NOE-restraints measured. This indicates that the structures are in agreement with both the orientational and distance restraints. It should be noted, however, that we do not want to put too much emphasis on the NOE restraints from $\mathrm{q}=0.5$ bicelles as we expect that the peptide-lipid interactions may be different for different lipid systems as

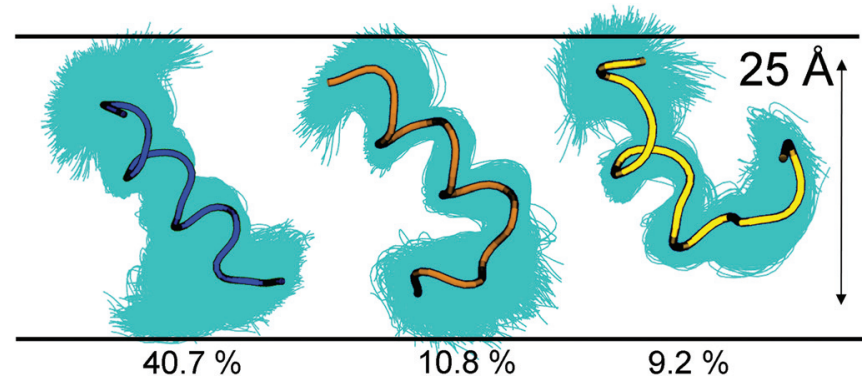

FIG. 10. Models selected from MD simulations which match the NMR data the best. The blue, orange, and yellow peptides are the median structures of clusters $1-3$, and the blue shadows in the background represent the individual structures in each cluster. The estimated position of the hydrophobic part of the bilayer is indicated in black lines. 


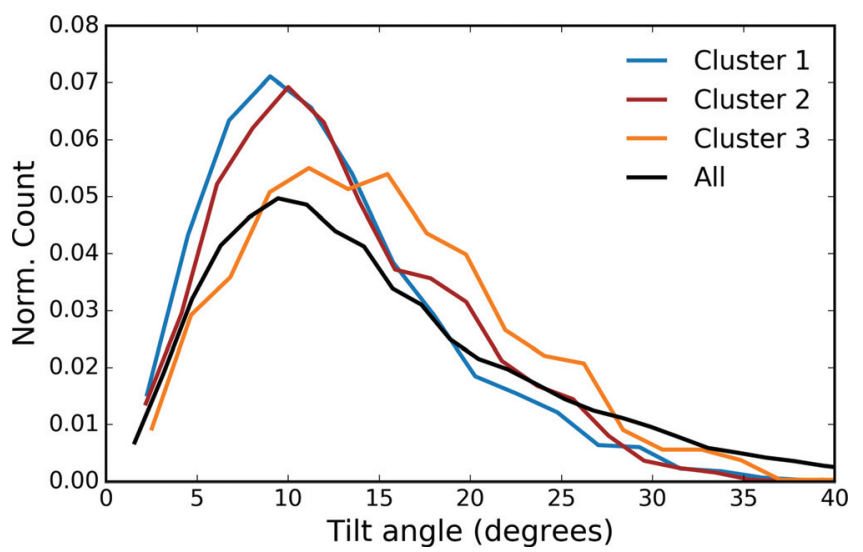

FIG. 11. Distributions of helix tilt angles of residues 2-7 of the peptides in the clusters and in all the selected models.

previously observed for alamethicin ${ }^{43}$ and as seen from the data above. It is apparent from Fig. 10 that mostly transmembrane peptides match the experimental data even though a significant fraction of the structures in the simulations were surface bound. The backbone angle distribution of the three clusters is shown Fig. S5 and reveals that cluster 1 is different from clusters 2 and 3 at $\operatorname{Asn} 2(\varphi)$ and $\operatorname{Leu11}(\varphi)$. Furthermore, all the clusters show backbone angles similar to that of Harzianin HC IX, with cluster 1 matching the best (Fig. S6). The central part of SPF (residues 3-10) thus shows a well-defined (Xaa-Yaa-Aib-Pro)- $\beta$-bend ribbon spiral conformation in all the selected conformations.

The selected peptides are consistently helical within residues 3-7, and this helix shows a tilted orientation with respect to the bilayer normal (Fig. 10). The distributions of helix tilt angles of this segment of the structures in the clusters are shown in Fig. 11. Clusters 1 and 2 seem to have very similar helix tilt angles of $9 \pm 5^{\circ}$ and $10 \pm 5^{\circ}$, respectively. Cluster 3 has a broader distribution with helix tilt angles of $12 \pm 7^{\circ}$. Looking at angle distribution of all the selected structures (black in Fig. 11), it is found that the most probable helix tilt angle is around $10^{\circ}$. A tilt angle of around $10^{\circ}$ is small, which is likely a result of the peptides being short compared to the hydrophobic thickness of the membrane as discussed in Sec. IV B.

\section{SUMMARY AND CONCLUSIONS}

The MD simulations showed that SPF spontaneously is inserted in a transmembrane orientation in lipid bilayers, which results in a more stretched conformation than that observed in the $\mathrm{q}=0.5$ bicelle experiments, thus adapting to the thickness of the bilayer. Additionally, bilayer thinning was observed near the peptide to obtain a better match between the length of the peptides and the hydrophobic thickness of the bilayer. This bilayer thinning could be what drives the aggregation of the peptides in the bilayer. Transition from a surface bound to the transmembrane orientation always occurred by the insertion of the C-terminal of the peptide as a result of it being much less hydrophilic than the N-terminal due to the special hydroxyl terminal group present instead of the usual carboxylate and the absence of hydrophilic residues. Long time scale coarse-grained simulations indicated that the peptides aggregate into two-layer bands. The transmembrane orientation was validated by scoring the peptide according to which conformations matched the NMR data from $\mathrm{q}=3.2$ bicelles the best. Finally, the combined effort of CD, NMR, and MD indicates that the secondary structure of SPF matches the (Xaa-Yaa-Aib-Pro)- $\beta$-bent ribbon spiral conformation previously observed for the homology peptide Harzianin HC IX. ${ }^{41}$ Thus, we propose that this may be the membrane bound conformation of all SPF-like peptides. The obtained knowledge explains the first steps of membrane perforation by SPF in atomic detail, and this information may be used to investigate the relationship between the amino acid sequence and the perforation mechanism as well as the structure of other helixlike conformation further. Future studies will include simulations on an even longer timescale to reveal more steps toward pore formation. This study demonstrates the power of combining NMR and MD simulations, and further development of combining NMR and MD will allow for an even better insight into the interplay between lipids and peptides.

\section{ACKNOWLEDGMENTS}

The authors thank the Danish National Research Foundation (DNRF 0059), the Danish Ministry of Higher Education and Science (AU-2010-612-181), the Lundbeck foundation (R191-2015-827), the Danish Council for Independent Research in Technology and Production (FTP 0602-01778b), the Sino-Danish Centre, and Aarhus University for financial support. Computations were made possible by the Centre for Scientific Computing Aarhus.

${ }^{1}$ M. Pasupuleti, A. Schmidtchen, and M. Malmsten, Crit. Rev. Biotechnol. 32, 143 (2012).

${ }^{2}$ G. Ehrenstein and H. Lecar, Q. Rev. Biophys. 10, 1 (1977).

${ }^{3}$ K. Matsuzaki, O. Murase, N. Fujii, and K. Miyajima, Biochemistry 35, 11361 (1996).

${ }^{4}$ Y. Pouny, D. Rapaport, A. Mor, P. Nicolas, and Y. Shai, Biochemistry 31, 12416 (1992).

${ }^{5}$ L. T. Nguyen, E. F. Haney, and H. J. Vogel, Trends Biotechnol. 29, 464 (2011).

${ }^{6}$ J. F. D. S. Daniel and E. Rodrigues Filho, Nat. Prod. Rep. 24, 1128 (2007).

${ }^{7}$ S. Rebuffat, C. Goulard, and B. Bodo, J. Chem. Soc., Perkin Trans. 1, 1849 (1995).

${ }^{8}$ B. Leitgeb, A. Szekeres, L. Manczinger, C. Vágvölgyi, and L. Kredics, Chem. Biodiversity 4, 1027 (2007).

${ }^{9}$ N. Hosotani, K. Kumagai, S. Honda, A. Ito, T. Shimatani, and I. Saji, J. Antibiot. 60, 184 (2007).

${ }^{10}$ P. K. Mukherjee, A. Wiest, N. Ruiz, A. Keightley, M. E. Moran-Diez, K. McCluskey, Y. F. Pouchus, and C. M. Kenerley, J. Biol. Chem. 286, 4544 (2011).

${ }^{11}$ R. Gessmann, D. Axford, R. L. Owen, H. Brückner, and K. Petratos, Acta Crystallogr. D 68, 109 (2012).

${ }^{12}$ M. Lucaciu, S. Rebuffat, C. Goulard, H. Duclohier, G. Molle, and B. Bodo, Biochim. Biophys. Acta Biomembr. 1323, 85 (1997).

${ }^{13}$ W. C. Wimley and S. H. White, Nat. Struct. Mol. Biol. 3, 842 (1996).

${ }^{14}$ H. F. Christoffersen, S. K. Hansen, B. S. Vad, E. H. Nielsen, J. T. Nielsen, T. Vosegaard, T. Skrydstrup, and D. E. Otzen, Biochim. Biophys. Acta 1854, 882 (2015).

${ }^{15}$ L. Pauling, R. B. Corey, and H. R. Branson, Proc. Natl. Acad. Sci. U. S. A. 37, 205 (1951). 
${ }^{16}$ N. Kučerka, M. Nieh, and J. Katsaras, Biochim. Biophys. Acta Biomembr. 1808, 2761 (2011).

${ }^{17}$ D. E. Shaw et al., Science 330, 341 (2010).

${ }^{18}$ S. K. Hansen, M. Vestergaard, L. Thøgersen, B. Schiøtt, N. C. Nielsen, and T. Vosegaard, J. Phys. Chem. B 118, 5119 (2014).

${ }^{19}$ L. Thøgersen, B. Schiøtt, T. Vosegaard, N. C. Nielsen, and E. Tajkhorshid, Biophys. J. 95, 4337 (2008).

${ }^{20}$ M. J. Abraham, T. Murtola, R. Schulz, S. Páll, J. C. Smith, B. Hess, and E. Lindahl, SoftwareX 1-2, 19 (2015).

${ }^{21}$ J. B. Klauda, R. M. Venable, J. A. Freites, J. W. O’Connor, D. J. Tobias, C. Mondragon-Ramirez, I. Vorobyov, A. D. MacKerell, and R. W. Pastor, J. Phys. Chem. B 114, 7830 (2010).

${ }^{22}$ S. Piana, K. Lindorff-Larsen, and D. Shaw, Biophys. J. 100, L47 (2011).

${ }^{23}$ E. Neria, S. Fischer, and M. Karplus, J. Chem. Phys. 105, 1902 (1996).

${ }^{24}$ H. J. C. Berendsen, J. P. Postma, W. F. van Gunsteren, A. DiNola, and J. R. Haak, J. Chem. Phys. 81, 3684 (1984).

${ }^{25}$ W. G. Hoover, Phys. Rev. A 31, 1695 (1985).

${ }^{26}$ M. Parrinello and A. Rahman, J. Appl. Phys. 52, 7182 (1981).

${ }^{27}$ U. Essmann, L. Perera, M. L. Berkowitz, T. Darden, H. Lee, and L. G. Pedersen, J. Chem. Phys. 103, 8577 (1995).

${ }^{28}$ P. J. Steinbach and B. R. Brooks, J. Comput. Chem. 15, 667 (1994).

${ }^{29}$ S. Miyamoto and P. A. Kollman, J. Chem. Theory Comput. 13, 952 (1992).

${ }^{30}$ B. Hess, H. Bekker, H. J. C. Berendsen, and J. G. E. M. Fraaije, J. Comput. Chem. 18, 1463 (1997).

${ }^{31}$ D. H. de Jong, G. Singh, W. F. D. Bennett, C. Arnarez, T. A. Wassenaar, L. V. Schäfer, X. Periole, D. P. Tieleman, and S. J. Marrink, J. Chem. Theory Comput. 9, 687 (2013).
${ }^{32}$ S. O. Yesylevskyy, L. V. Schäfer, D. Sengupta, and S. J. Marrink, PLoS Comput. Biol. 6, e1000810 (2010).

${ }^{33}$ L. Monticelli, S. K. Kandasamy, X. Periole, R. G. Larson, D. P. Tieleman, and S. Marrink, J. Chem. Theory Comput. 4, 819 (2008).

${ }^{34}$ S. J. Marrink, A. H. de Vries, and A. E. Mark, J. Phys. Chem. B 108, 750 (2004).

${ }^{35}$ G. Bussi, D. Donadio, and M. Parrinello, J. Chem. Phys. 126, 014101 (2007).

${ }^{36}$ T. A. Wassenaar, K. Pluhackova, R. A. Böckmann, S. J. Marrink, and D. P. Tieleman, J. Chem. Theory Comput. 10, 676 (2014).

${ }^{37}$ A. C. Dahl, M. Chavent, and M. S. Sansom, Bioinformatics 28, 2193 (2012).

${ }^{38}$ V. Gapsys, B. de Groot, and R. Briones, J. Comput.-Aided Mol. Des. 27, 845 (2013).

${ }^{39}$ S. K. Hansen, K. Bertelsen, B. Paaske, N. C. Nielsen, and T. Vosegaard, Prog. Nucl. Magn. Reson. Spectrosc. 88-89, 48 (2015).

${ }^{40}$ N. Kučerka, Y. Liu, N. Chu, H. I. Petrache, S. Tristram-Nagle, and J. F. Nagle, Biophys. J. 88, 2626 (2005).

${ }^{41}$ I. Ségalas, Y. Prigent, D. Davoust, B. Bodo, and S. Rebuffat, Biopolymers 50, 71 (1999).

${ }^{42}$ M. Vestergaard, J. F. Kraft, T. Vosegaard, L. Thøgersen, and B. Schiøtt, J. Phys. Chem. B 119, 15831 (2015).

${ }^{43}$ K. Bertelsen, B. Vad, E. H. Nielsen, S. K. Hansen, T. Skrydstrup, D. E. Otzen, T. Vosegaard, and N. C. Nielsen, J. Phys. Chem. B 115, 1767 (2011).

${ }^{44}$ See supplementary material at http://dx.doi.org/10.1116/1.4982642 for details about the experimental procedure and supplementary figures. 